\title{
Development of sensor system for real-time measurement of droplet deposition of agricultural sprayers
}

\author{
Longlong $\mathrm{Li}^{1,4}$, Ruirui Zhang ${ }^{1,4}$, Liping Chen ${ }^{2,3^{*}}$, Tongchuan $\mathrm{Yi}^{2,3}$, Gang $\mathrm{Xu}^{1,4}$, \\ Daxuan Xue ${ }^{5}$, Qing Tang ${ }^{2,3}$, Linhuan Zhang ${ }^{1,4}$, Andrew John Hewitt ${ }^{6}$, Yining An $^{7}$ \\ (1. Beijing Research Center of Intelligent Equipment for Agriculture, Beijing Academy of Agricultural and Forestry Sciences, \\ Beijing 100097, China; \\ 2. National Research Center of Intelligent Equipment for Agriculture, Beijing 100097, China; \\ 3. National Center for International Research on Agricultural Aerial Application Technology, Beijing 100097, China; \\ 4. Beijing Key Laboratory of Intelligent Equipment Technology for Agriculture, Beijing Academy of Agricultural and Forestry Sciences, \\ Beijing 100097, China; \\ 5. College of Information Engineering, Capital Normal University, Beijing 100048, China; \\ 6. Centre for Pesticide Application and Safety, the University of Queensland, Queensland 4072, Australia; \\ 7. Faculty of Science and Engineering, the University of Nottingham Ningbo China, Ningbo 315100, Zhejiang, China)
}

\begin{abstract}
During the chemical application process, droplet deposition on a target is an important reference indicator for evaluating the spraying technique and its performance. In order to quickly obtain deposition results in the field, this study proposed a novel system based on surface humidity sensors. The basic principle is to convert the measured physical quantity change into a capacitance change, thereby realizing the physical quantity to electrical signal conversion. An Android application for mobile terminal and the corresponding coordinator were developed, which allowed operators to control multiple sensors simultaneously through the Bluetooth. The soluble tracer detected by spectrophotometer was used to calibrate the system. The obtained results indicated a good correlation between deposition volume and voltage increment output from the newly developed system ( $R^{2}$ of the six nozzles with $D_{v 0.5}$ ranging from $107.28 \mu \mathrm{m}$ to $396.20 \mu \mathrm{m}$ were $\left.0.8674-0.9729\right)$, and a power regression model based on the least squares technique $\left(R^{2}=0.8022\right)$ was developed. In the field test, the system exhibited an optimal performance in predicting the deposition volume. Compared with the conventional method of measuring tracer concentration, the deviation was less than $10 \%$. In addition, the system exhibited good fitting curve of the deposition distribution with droplet number results measured by the water sensitive paper method.
\end{abstract}

Keywords: deposition measurement, agricultural sprayer, droplets, intelligent sensor

DOI: $10.25165 /$ j.ijabe.20211405.5528

Citation: Li L L, Zhang R R, Chen L P, Yi T C, Xu G, Xue D X, et al. Development of sensor system for real-time measurement of droplet deposition of agricultural sprayers. Int J Agric \& Biol Eng, 2021; 14(5): 19-26.

\section{Introduction}

During the chemical application process of agricultural sprayers, droplet deposition characteristics on the target mainly include distribution uniformity and deposition volume, which are direct indicators for testing the control effect of chemical liquids ${ }^{[1-3]}$.

Received date: 2019-11-18 Accepted date: 2021-06-08

Biographies: Longlong $\mathbf{L i}, \mathrm{PhD}$, research interests: plant protection machinery and application technology, Email: lill@ nercita.org.cn; Ruirui Zhang, research interests: agricultural aviation application and precise spraying, Email: zhangrr@nercita.org.cn; Tongchuan Yi, Engineer, research interests: electrics and electronics, Email: yitc@nercita.org.cn; Gang Xu, PhD, research interests: image processing and information acquisition, Email: xug@nercita.org.cn; Daxuan Xue, Master candidate, research interests: software engineering, Email: 405529829@qq.com; Qing Tang, PhD, research interests: agricultural aviation application, Email: tangq@nercita.org.cn; Linhuan Zhang, PhD, research interests: precision agriculture, Email: zhanglh@nercita.org.cn; Andrew John Hewitt, Professor, research interests: agricultural aviation application, Email: a.hewitt@uq.edu.au; Yining An, research interests: microelectronics and sensing, Email: shyya1@nottingham.edu.cn.

*Corresponding author: Liping Chen, Professor, research interests: intelligent agricultural technology, precision agriculture. Beijing Nongke Mansion, No. 11 Shuguang Huayuan Middle Road, Haidian District, Beijing 100097, China. Tel: +86-10-51053425, Email: chenlp@nercita.org.cn.
The primary objective in all spray applications is to obtain adequate coverage and uniform pesticide deposition on the target, to provide sufficient effectiveness against pest ${ }^{[4,5]}$. In order to improve spray application techniques, it is important to measure and quantify the spray deposition and distribution ${ }^{[6]}$. However, environmental conditions, application parameters, application machinery and other factors may lead to poor droplet deposition, which affects the later biological control effect. The operator needs to re-spray or take other remedial measures to improve droplet deposition based on the results of the spraying test. Therefore, fast and accurate droplet deposition detection can provide more efficient data support for agricultural or scientific practitioners to make better chemical application decisions or spray quality evaluations ${ }^{[7,8]}$.

Hence, various methods have been developed to obtain droplet deposition during the spray process. The magnesium oxide method adopts the principle of droplet color development to determine droplet deposition characteristics. A magnesium strip was burned on a glass slide to obtain a magnesium oxide coating. When droplets contact with magnesium oxide, a circular mark is formed ${ }^{[9]}$. A few researchers have used silicone oil to obtain colored droplets based on the density of the two liquids ${ }^{[10]}$. Generally, these two methods are cumbersome to operate and have a large workload. Furthermore, they have been gradually 
eliminated in addition to a few special tests. Chemical tracers have been widely adopted for pesticide spray deposition assessment to simulate the deposition of pesticides ${ }^{[11]}$. The analytical methods used to determine tracer concentration based on absorbency and spectral intensity, commonly used tracers include brilliant sulfoflavine ${ }^{[12]}$, tartrazine ${ }^{[13]}$, erythrosine ${ }^{[14]}$, pyranine ${ }^{[15]}$. Usually, passive collectors such as Petri dishes, filter paper and mylar card are placed in the treated area as artificial targets. When spraying is completed, the tracer is recovered by deionized water and its concentration is determined by spectrophotometer or fluorimeter. Some authors have reported the chemical tracers will degrade when exposed to sunlight, and the recovery rate of tracers recovered from artificial or natural targets affects the droplet deposition result ${ }^{[16]}$. To address these limitations of chemical tracers, metallic salt tracers have been applied by researchers in field trials ${ }^{[17]}$. This method does not degrade when exposed to solar radiation compared with synthetic chemical tracers, and as a natural product, it is perceived as less environmentally damaging.

Water sensitive paper (WSP) card was developed approximately 30 years ago. The cards have been widely used by farmers and researchers to obtain deposition parameters such as spray coverage rate, droplet density, and droplet number ${ }^{[18]}$. This method mainly applies the image analysis technology to read densely stained targets. Compared with quantitative methods for measuring tracer concentration, WSP provides a fast and inexpensive method for spray deposition assessment. However, previous studies indicated that the yellow coating could return blue in a high-humidity environment, and small droplet less than $50 \mu \mathrm{m}$ cannot produce detectable stains on the coating ${ }^{[19]}$. As a result, this method is limited in some scenarios. At present, WSP is more often applied to field demonstrations or assessing the spraying quality.

As aforementioned, the techniques for detecting spray deposition can effectively obtain the deposition amount; however, there are several limitations of their application, which include the fact that it is time-consuming, operation-complicated and laborious ${ }^{[20]}$. In addition, the deposition results infield cannot be calculated in real time, but can only be measured under laboratory conditions.

With the development of smart agricultural technology, modern technical means are increasingly being applied in agriculture. In the previous works on spraying tests, researchers established a method for measuring droplet deposition on the ground based on infrared spectroscopy and spectral reflectance rate technology. Salyani et al. ${ }^{[21]}$ developed a deposition sensor based on the principle of variable resistors, and a model of the relationship between the output voltage of the sensor and the droplet deposition was established. Zhang et al. ${ }^{[22]}$ designed an aerial deposition volume detection system based on spectral analysis combined with the fluorescence excitation technology, which can obtain the continuous distribution characteristics of droplet deposition. These methods enable fast and efficient acquisition of the droplet deposition. However, owing to the limitations of the design, most of the testing equipment remain in the experimental stage and cannot be widely used.

To address these limitations, an intelligent sensor system for the real-time measurement of droplet deposition (ISSRMDP) for agricultural sprayers was developed. The main objectives of this research were: 1) to develop a simple and reliable deposition indicator with wireless sensor networks (WSNs) and 2) to test and validate the novel system for real-time measurements.

\section{Materials and methods}

\subsection{Electro-leaf sensor}

A surface humidity sensor with a leaf shape was adopted as the sensitive device (HSTL-YM10, Beijing Huakongxingye Inc., China). The basic principle is to convert the measured physical quantity change into a capacitance change to realize the measurement. The sensor has the advantages of simple structure, high resolution, high temperature resistance and radiation resistance, and it can work stably in the field, under harsh conditions. The characteristic parameters are presented in Table 1.

Table 1 Characteristics parameters of the electro-leaf sensor used in the study

\begin{tabular}{lc}
\hline \multicolumn{1}{c}{ Items } & Values \\
\hline Output voltage/V & $0-2$ \\
Response time/s & $<1$ \\
Power consumption $/ \mathrm{W}$ & 0.22 \\
Storage temperature $/{ }^{\circ} \mathrm{C}$ & $-40-85$ \\
Protection grade & IP 65 \\
Size $/ \mathrm{mm} \times \mathrm{mm} \times \mathrm{mm}$ & $65 \times 13 \times 145$ \\
\hline
\end{tabular}

\subsection{Principle of deposition measurement}

The structure of the sensor is presented in Figure 1a. The surface is distributed with multiple parallel rows of variable dielectric constant capacitors with equal spacing. As illustrated in Figure $1 \mathrm{~b}, \mathrm{~A}$ and $\mathrm{B}$ depict metal plates with a certain spacing $d$, thickness $t$, and length $L$. Capacitors are fixed on the resin board and the surface is sprayed with an insulating coating.

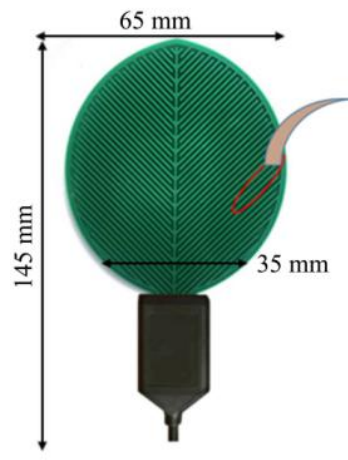

a. Sensor structure

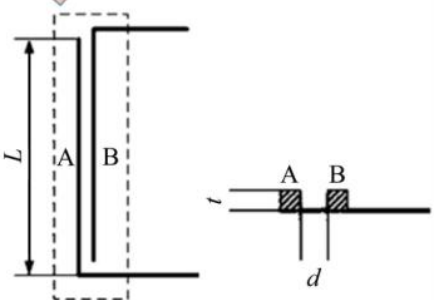

b. Capacitor structure
Note: A and B depict metal plates; $L, d$, and $t$ represent the length, distance between metal plates, and thickness of the metal plates, respectively.

Figure 1 Sketch diagram of working principle of electro-leaf sensor

In the absence of droplet deposition, the dielectric between the two plates of the capacitor on the surface of the sensor is air, and the capacitance $C_{0}$ of the capacitor is calculated as:

$$
\begin{gathered}
C_{0}=\frac{\varepsilon_{0} S_{0}}{d} \\
S_{0}=L t
\end{gathered}
$$

where, $C_{0}$ is capacitance, $\mathrm{F}$; $\varepsilon_{0}$ is air's dielectric constant, $\mathrm{F} / \mathrm{m} ; S_{0}$ is the covered area of metal plates, $\mathrm{m}^{2} ; d$ is the distance between metal plates, $\mathrm{m} ; t$ is the thickness of metal plates, $\mathrm{m} ; L$ represents the length, $\mathrm{m}$.

When spray droplet deposition is retained by the leaf sensor surface, the dielectric between the two plates of the capacitor becomes a mixture of air and droplets. Because the dielectric constant of the droplet differs from that of air, the dielectric constant between the two plates of the capacitor changes. The spacing and thickness of the capacitor plates are small, and the 
covered area $S_{0}$ between the capacitor plates is divided into the air-covered $S_{1}$ and the droplet-covered $S_{2}$. The dielectric constant of the chemical solution was set as $\varepsilon_{\mathrm{d}}$. According to Equation (1), the capacitor capacitance $C_{\mathrm{d}}$ of the droplet deposition can be expressed as:

$$
\begin{gathered}
C_{\mathrm{d}}=\frac{\varepsilon_{0} S_{1}+\varepsilon_{\mathrm{d}} S_{2}}{d}=\frac{\varepsilon_{0}\left(S_{0}-S_{2}\right)+\varepsilon_{\mathrm{d}} S_{2}}{d}=\frac{\varepsilon_{0} S_{0}}{d}+\frac{\left(\varepsilon_{\mathrm{d}}-\varepsilon_{0}\right) S_{2}}{d} \\
=C_{0}+\frac{\left(\varepsilon_{\mathrm{d}}-\varepsilon_{0}\right) S_{2}}{d} \\
C_{\mathrm{d}}=C_{0}=\frac{\left(\varepsilon_{\mathrm{d}}-\varepsilon_{0}\right) S_{2}}{d}
\end{gathered}
$$

During the spray application process, once the concentration of the sprayed solution was fixed, the dielectric constant of the spraying solution was constant when the influence of ambient temperature was neglected. Therefore, it is evident that the capacitance change $C_{\mathrm{d}}-C_{0}$ is linearly related to the univariate of the droplet coverage area $S_{2}$. In order to obtain an electrical signal that can be easily detected, the capacitance is converted into a voltage signal (Figure 2). Finally the change in the voltage output can be used to reflect the change in the deposition coverage area of the droplet on the sensor.

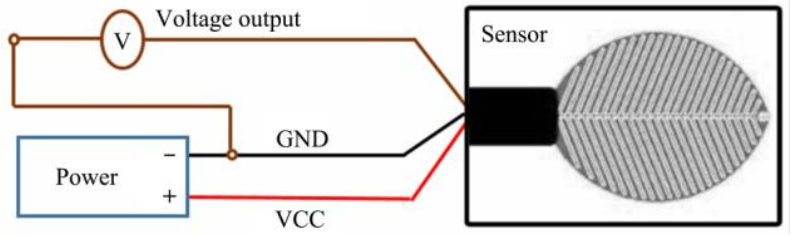

Figure 2 Schematic diagram of electro-leaf sensor connection

\subsection{Development of ISSRMDP}

\subsubsection{Description of the system}

The system primarily comprises three parts: sensor nodes, coordinator and mobile phone terminal (Figure 3). The coordinator and sensor nodes rely on the ZigBee information technology for signal transmission, and the mobile terminal and coordinator transmit the command signal via Bluetooth. In order to meet the multi-region layout requirements, the system can connect multiple sensor nodes for simultaneous deposition measurement. Before the system was applied, each sensor node was encoded and stored in the coordinator and mobile terminal, and the data were guaranteed to correspond to the sensor nodes using the transmission protocol. Once the system equipment was deployed and worked, the sensor nodes and coordinator self-organized to form a Zigbee and tree topology network according to the network size and the distance between the coordinator and sensor nodes. Deposition data obtained by the sensor in the network was sent to the coordinator. The coordinator sent data to the mobile phone via Bluetooth, and mobile phone could also send command and configuration information to the sensor nodes via the coordinator (Figure 4).

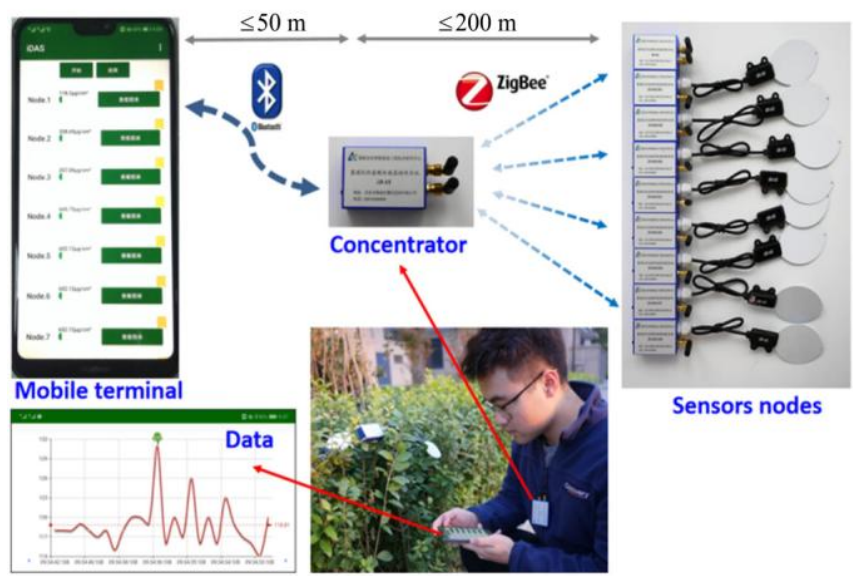

Figure 3 Diagram of the overall composition of ISSRMDP
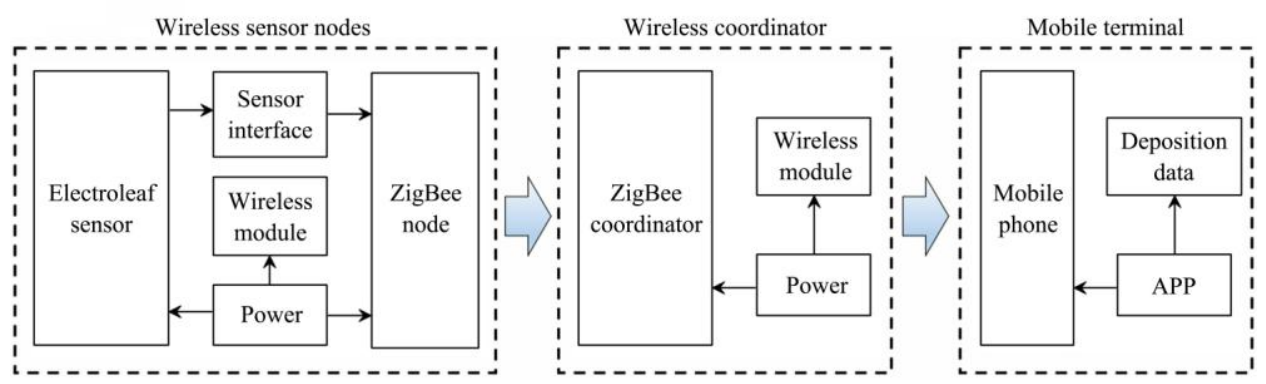

Figure 4 Schematic diagram of work flow of the sensor system

\subsubsection{System design}

The sensor nodes primarily comprised a circuit board, radio frequency communication antenna, leaf sensor and power circuit (Figure 5a). An atmega 168 PA-AU microcontroller (Yunhui Inc., China) processed the data and commands. To realize data acquisition and transmission, a wireless circuit with CC 2530 chip (WeBee Inc., China) was developed. The deposition information was converted into a voltage of $0-2000 \mathrm{mV}$ by a leaf sensor, and the sensor node acquired the voltage signal at a fixed frequency of $2 \mathrm{~Hz}$. To improve data accuracy, the amplified circuit with an AD822 amplifier (Xinwei, Inc., China) and a 10-bit analog-digital converter was designed. During the test, the deposition results were temporarily stored in the microcontroller, and waited for the data query signal. When the data query signal was received, the data was sent to the coordinator by CC 2530 chip.

Sensor nodes operated at a voltage of $3.3 \mathrm{~V}$. The system was powered by a lithium battery with a full voltage of $4.2 \mathrm{~V}$, which was converted to $3.3 \mathrm{~V}$ by a linear voltage regulator. In general, we assume that when the battery voltage is below $3.6 \mathrm{~V}$, the system will be in a low charge state, indicating that it needs to be charged. Also, the system supports the use of $5 \mathrm{~V}$ mobile power supply instead of battery when working long hours in the field.

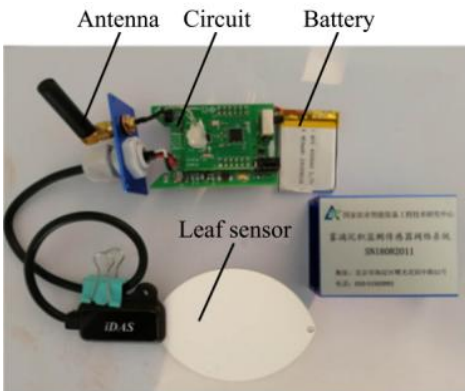

a. Wireless sensor node

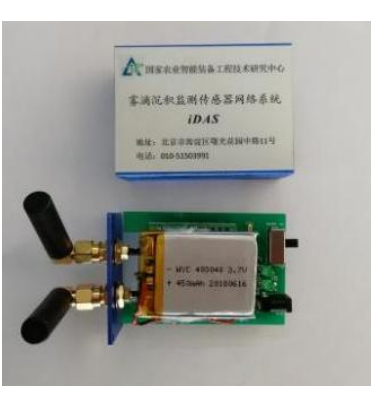

b. Wireless coordinator
Figure 5 Hardware circuit of sensor system

The wireless coordinator was responsible for the transfer and distribution of data (Figure 5b). It was connected to the mobile 
terminal using the HC-06 Bluetooth device, and data interaction performed with the wireless sensor node using the CC 2530 chip. HC-06 and CC 2530 chips were serially exchanged at 38400 baud through an onboard circuit.

2.3.3 Android application for mobile terminal

A virtual interface (VI) built in Java language (IntelliJ IDEA Version2018.2, JetBrains, Co., Czech Republic) was used to measure, process, control, compensate, and record the data collected from peripheral devices. In the working process, the Bluetooth node is connected by a Bluetooth adapter that comes preinstalled with android system. When the connection was successful, the hexadecimal data of the virtual voltage signals representing deposition volume were transmitted to the application by the Bluetooth. To facilitate further processing of the data, thread class technology was applied to monitor the data in real-time, and the acquired data could be saved in the mobile terminal through the data access object mechanism. Also, to avoid excessive memory consumption of the mobile phone, a data-clearing function was added to the system.

\subsection{Testing of ISSRMDP}

\subsubsection{Laboratory analysis}

As mentioned above, when droplets are deposited on leaf sensor surface, the system outputs different voltage signals. To explore the relationship between actual droplet deposition on the sensor and output signal from the sensor, a test was carried out in laboratory.

1) Experiment set up and spray application approach

The experiments were conducted in agricultural aerial application laboratory at the Beijing Research Center of Intelligent Equipment for Agriculture. The layout of the test site is presented in Figure 6. The nozzle was fixed at a height of $1.0 \mathrm{~m}$, and the leaf sensor was placed $0.5 \mathrm{~m}$ below the nozzle. Six types of nozzles widely used in agriculture were applied in the test: air-injector flat spray compact nozzles IDK120-03 and IDK120-04, standard flat spray nozzle ST110-08, and hollow cone nozzles TR80-03, TR80-04 and TR80-005. The spray time was set to 100 to $2580 \mathrm{~ms}$, at an interval of $80 \mathrm{~ms}$. In order to control the spray time accurately, a timing switch was designed to control the solenoid valve. When the spray time was reached, the timing switch was de-energized and the solenoid valve closed. At this point, the nozzle stopped spraying. To elucidate the stability of the liquid transport in real-time during the test, a flow meter and pressure gauge were installed on the upstream pipeline of the nozzle. The spraying pressure was set to $0.35 \mathrm{MPa}$ and the spray mixture containing $1.5 \mathrm{~g} / \mathrm{L}$ water-soluble food dye (Ponceau 4R, Shanghai Dyestuffs Research Institute Co., Ltd. Shanghai, China) was adopted as the tracer. Each treatment combination was repeated three times, and a total of 576 sets of tests were conducted.

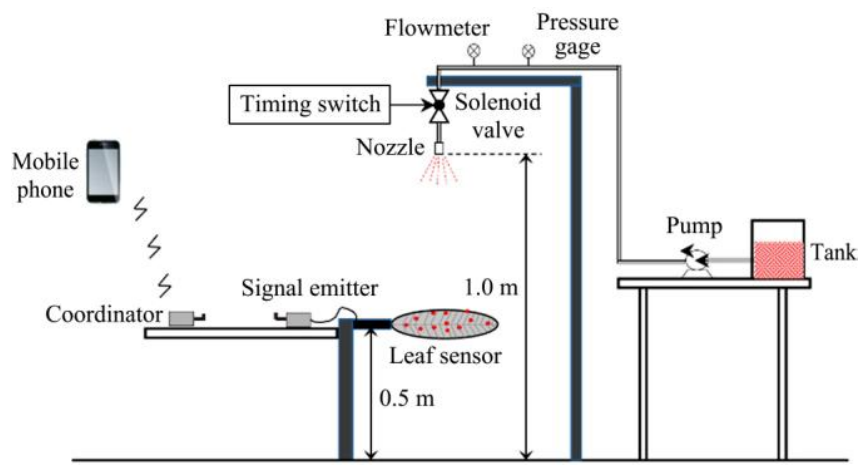

Figure 6 Schematic diagram of deposition test platform
2) Determination of tracer deposition

Once a trial was completed, mobile terminal recorded the voltage, and the tracer droplets on the leaf sensor were washed with $20 \mathrm{~mL}$ of distilled water in a ziplock bag. To enable the tracer to be completely eluted from the sensor surface, a pipette with a range of $10 \mathrm{~mL}$ was used to remove the deionized water. This method is easy to control the water flow rate artificially when using a fixed volume of deionized water to completely elute the tracer on sensor surface. The absorbance of the extracts was measured using a spectrophotometer (752N INESA, Shanghai, China) at a wavelength of $508 \mathrm{~nm}^{[23]}$. The results were obtained in terms of surface deposit, as defined by Equation (5) ${ }^{[24]}$ :

$$
\beta_{\text {dep }}=\frac{\left(A b s_{\text {samp }}-A b s_{\text {blk }}\right) \times V_{\text {dil }} \times 10^{3}}{A b s_{\text {spray }}}
$$

where, $\beta_{\text {dep }}$ is the deposition volume on the sensor surface, $\mu \mathrm{L}$; $A b s_{\text {samp }}$ is the spectrophotometer absorbance value of the sample; $A b s_{b l k}$ is the absorbance reading of the blanks; $V_{d i l}$ is the volume of dilution liquid used to solute the tracer from sensor surface, $\mathrm{mL}$; $A b s_{\text {spray }}$ is the spectrophotometer absorbance value of mixture in tank.

\section{3) Measurement of droplet diameter}

The droplet size measurements of six types of nozzles used in the experiment were performed using a droplet size distribution analyzer equipped with a laser diffraction system (HELOS-VARIO/KR, SYMPATEC Co., Ltd, Germany). The transmitter and receiver were adjustable in the range of 123$1400 \mathrm{~mm}$. In this test, the distance between the transmitter and receiver was set as $1200 \mathrm{~mm}$. The device was equipped with seven lenses (R1 R7) and can be selected according to the droplet size range. The R7-lenses were selected because of their large measuring range from $0.5 \mu \mathrm{m}$ to $3500 \mu \mathrm{m}$. The data acquisition time was $15 \mathrm{~s}$. The nozzle was installed above the center of the transmitter and receiver, and the distance between the lower end of nozzle and the laser beam was $0.5 \mathrm{~m}$. For flat spray nozzles (IDK120-03, IDK120-04 and ST110-08), the fan atomized spraying plane was perpendicular to the laser beam. The pressure was $0.35 \mathrm{MPa}$, and the spraying time was set to $20 \mathrm{~s}$. Each treatment combination was repeated three times. In the experiment, $D_{v 0.1}, D_{v 0.5}$ and $D_{v 0.9}$ were measured, and relative span factor $(R S F)$, which represents dimensionless indicator of the uniformity of the drop size distribution was calculated according to Equation (6) ${ }^{[25]}$ :

$$
R S F=\frac{D_{v 0.9}-D_{v 0.1}}{D_{v 0.5}}
$$

where, $R S F$ is the relative span factor; $D_{v 0.1}, D_{v 0.5}$ and $D_{v 0.9}$ is the maximum droplet diameter below which $10 \%, 50 \%$ and $90 \%$ of the volume of the sample exists, respectively, $\mu \mathrm{m}$.

2.4.2 Field test

Field experiments were conducted at National Precision Agriculture Research Center in Changping district of Beijing, China. To access the performance of the sensor system, a single-rotor plant protection agricultural unmanned aerial vehicle (UAV, TTA-T8-PRO-5, Anyang Quanfeng aviation plant protection technology Co., Ltd) was considered in this study. The UAV was powered by gasoline, and the maximum load was $10 \mathrm{~L}$. The operating parameters of the UAV are presented in Table 2. Six wireless sensor nodes (S1-S6) were mounted on a bracket with an interval of $0.5 \mathrm{~m}$ at the height of $0.7 \mathrm{~m}$ above the ground, and the total sample distance was $2.5 \mathrm{~m}$ (Figure 7). Six WSP cards $(26 \mathrm{~mm} \times 76 \mathrm{~mm})$ were attached next to the corresponding coded 
leaf sensor. Ponceau 4R solution with a concentration of $5.0 \mathrm{~g} / \mathrm{L}$ was adopted as spraying liquid.

Table 2 Spraying parameters of UAV used in experiment

\begin{tabular}{lc}
\hline \multicolumn{1}{c}{ Items } & Values \\
\hline UAV type & TTA-T8-PRO-5 \\
Spraying equipment & PLD-1206 \\
Flow rate $/ \mathrm{L} \cdot \mathrm{min}^{-1}$ & 0.75 \\
Flight height $/ \mathrm{m}$ & 2.5 \\
Spraying $\mathrm{swath} / \mathrm{m}$ & 3 \\
Flight speed $/ \mathrm{m} \cdot \mathrm{s}^{-1}$ & 2 \\
\hline
\end{tabular}

When one trial was completed, the deposition data from six sensors were recorded by mobile phone, and the tracer on sensor surface was eluted into a ziplock bag with $20 \mathrm{~mL}$ deionized water. . Then droplet deposition on sensor surface was determined according to the spectrophotometer mentioned above. WSP cards were scanned by a portable business card scanner (V8, Hanvon Technology Co., Ltd., China) with an imaging resolution of 600 dpi. These images were processed with "DepositScan" software developed by the USDA-ARS ${ }^{[26]}$. Finally, droplet number per unit area and deposit coverage rate were calculated and saved.

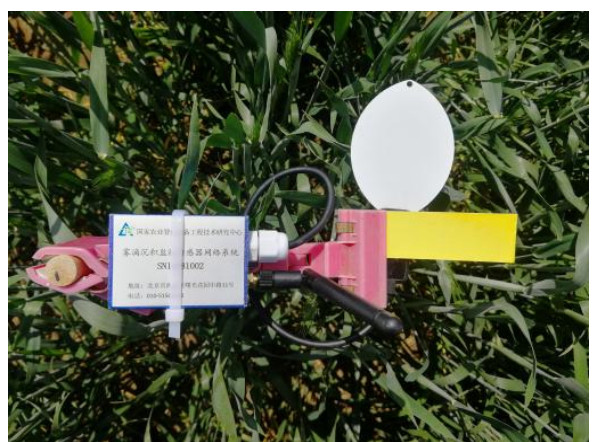

a. Leaf sensor and water sensitive paper at each sampling position

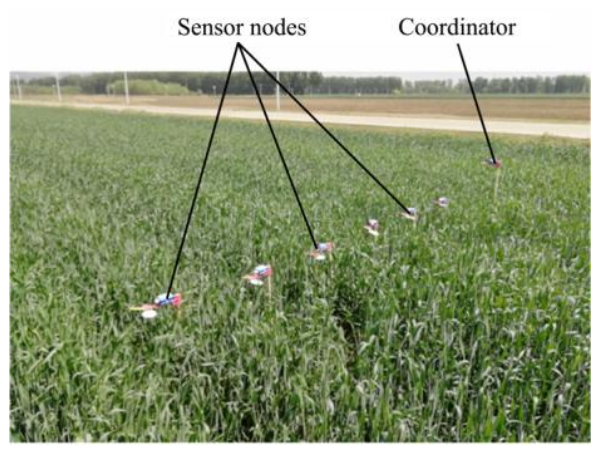

b. Layout of the sensor system

Figure 7 Water sensitive paper and sensor system used to measure droplet deposition

\section{Results and discussion}

A novel sensor system, ISSRMDP, was designed and fabricated for the real-time measurement of droplet deposition. The system was tested to determine the correlation between deposition amount and voltage output from leaf sensor, and to check the influence of droplet size from different agricultural nozzles on sensor system output signals. In addition, field tests were performed with an agricultural UAV.

\subsection{Laboratory analysis}

\subsubsection{Droplet diameter of different nozzles}

The droplet diameters of six types of agricultural nozzles were obtained in pesticide application testing laboratory of the Beijing
Research Center of Intelligent Equipment for Agriculture. After each test, the data including $D_{v 0.1}, D_{v 0.5}, D_{v 0.9}$ and $R S F$ were obtained using a specific software (WX5-RODOS, HELOS-VARIO/KR, SYMPATEC Co., Ltd, Germany). The droplet diameter parameters $D_{v 0.1}, D_{v 0.5}$ and $D_{v 0.9}$ for every nozzle type were recorded, calculated, and presented in Table 3.

Among the various parameters characterizing the range of droplet sizes during spraying, the most commonly used is the volumetric median diameter $\left(D_{v 0.5}\right)$. It can be deduced from Table 3 that the mean value of $D_{v 0.5}$ for nozzle TR80-005 is the smallest $(107.28 \mu \mathrm{m})$. The mean $D_{v 0.5}$ of nozzles TR80-03, TR80-04, ST110-08 are 136.93, 168.81 and $207.51 \mu \mathrm{m}$, respectively. The IDK type nozzle was designed with air induction technology to generate larger droplets and reduce the potential drift, thus, the $D_{v 0.5}$ of IDK120-03 and IDK120-04 are significantly higher than those of the other nozzles, and its maximum size is $396.20 \mu \mathrm{m}$. For all six types of nozzles used in this study, the range of droplet size was $100-400 \mu \mathrm{m}$, which could basically cover the size of most commonly used nozzles in agriculture. Based on the droplet diameter, it is possible to test applicability of ISSRMDP designed within the usual droplet size. In the case of $R S F$, the smaller the $R S F$, the narrower the droplet size distribution. This indicates that there are more droplets with diameters approximate to $D_{v 0.5}$. The results demonstrated that the maximum $R S F$ of the nozzles tested in this study was lower than 2.02, which suggests that the selected nozzles exhibited an optimal atomization effect.

Table 3 Measured diameter parameters of six agricultural nozzle types

\begin{tabular}{ccccc}
\hline \multirow{2}{*}{ Nozzles } & \multicolumn{4}{c}{ Droplet size characteristic parameters } \\
\cline { 2 - 5 } & $D_{v 0.1} / \mu \mathrm{m}$ & $D_{v 0.5} / \mu \mathrm{m}$ & $D_{v 0.9} / \mu \mathrm{m}$ & $R S F$ \\
\hline TR80-005 & 51.58 & 107.28 & 173.50 & 1.13 \\
TR80-03 & 56.63 & 136.93 & 292.44 & 1.72 \\
TR80-04 & 62.35 & 168.81 & 403.92 & 2.02 \\
ST110-08 & 79.94 & 207.51 & 463.37 & 1.84 \\
IDK120-03 & 155.79 & 372.57 & 654.36 & 1.34 \\
IDK120-04 & 156.31 & 396.20 & 698.19 & 1.36 \\
\hline
\end{tabular}

3.1.2 Influence of droplet size on ISSRMDP output signal

When droplets are deposited on the sensor surface, the system outputs a voltage signal. Hence, it is important to convert the voltage signal into a deposition value. Meanwhile, multiple electrodes are evenly distributed on sensor's surface, and the distance between the electrodes is fixed, thus, the droplet size may affect the accuracy of the output signal. Figure 8 presents the droplet deposition measured by a spectrophotometer and the voltage increment output from ISSRMDP. Evidently, for nozzles TR80-03, TR80-04, TR80-005, ST110-08 and IDK120-03 with $D_{v 0.5}$ ranging from $107.28 \mu \mathrm{m}$ to $372.57 \mu \mathrm{m}$, there were good correlations $\left(R^{2}=0.9035\right.$ to 0.9729$)$ between the two methods in measuring deposition on sensor's surface. Among the five nozzles, the nozzle TR80-04 and ST 110-08 exhibit the best correlation (Figure $8 \mathrm{~b}$ and $8 \mathrm{~d}, R^{2}=0.9707$ and 0.9729 , respectively), and the $D_{v 0.5}$ of the two nozzles are $168.81 \mu \mathrm{m}$ and $207.51 \mu \mathrm{m}$, respectively. In the range of $D_{v 0.5}$ mentioned above, droplet size had no significant effect on the correlation between the two methods. Nozzle IDK120-04 exhibits a lower correlation (Figure 8f, $\left.R^{2}=0.8674\right)$ between the tracer deposition and voltage increment. This discrepancy may be attributed to the adhesion between the large-size droplets. If the droplets are too large, the adhesion between the droplet increases, and consequently affect the 
total area $S_{0}$ between the plates (Equation (2)). Therefore, the voltage of the sensor output generates random fluctuations, resulting in some error. In general, these results indicate a strong correlation between actual deposition and electrical signals of

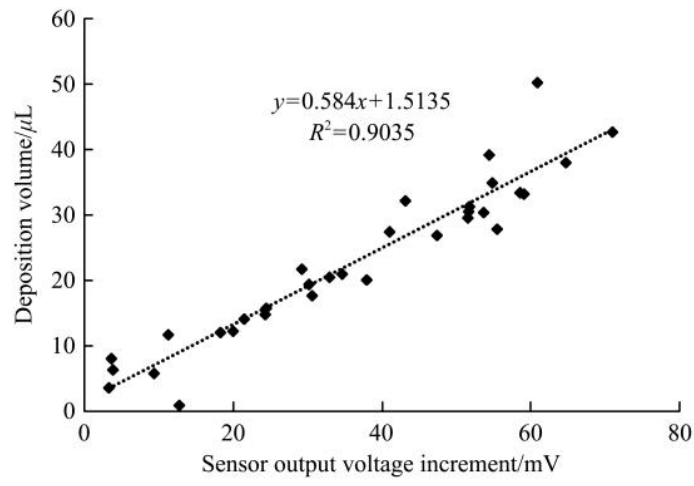

a. TR80-03 $\left(D_{v 0.5}=136.93 \mu \mathrm{m}\right)$

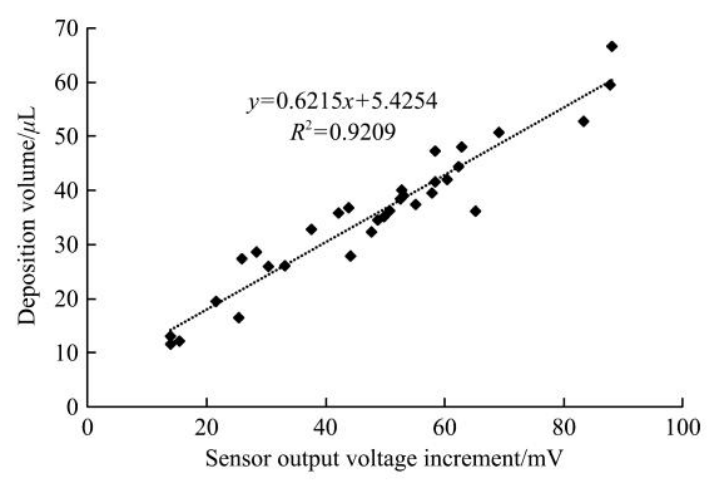

c. TR80-005 $\left(D_{v 0.5}=107.28 \mu \mathrm{m}\right)$

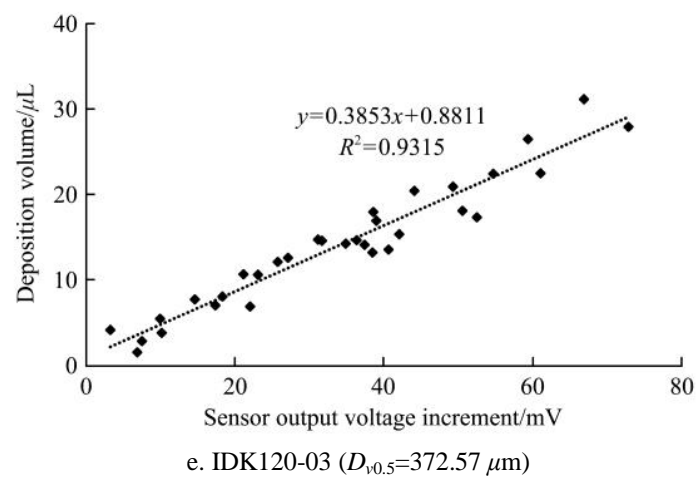

Figure 8 Relationship between deposition and voltage increment obtained by spectrophotometer and ISSRMDP, respectively

\subsubsection{Development of calibrating model}

Usually the sizes of droplets that spout from a nozzle are not completely consistent. As shown in Table 3, the RSF of the nozzles used in the experiment are different. For example, for nozzle TR80-04, the RSF is 2.02, but that of nozzle IDK120-03 is 1.34. Also, in actual spraying process, the types of nozzles applied vary according to chemical kinds and application technology. Hence, it is necessary to establish a calibration model to meet the size range measurement requirements. Based on the results presented in section 3.1.2, all the data were analyzed.

A power regression based on the least squares technique was adopted to convert the sensor's electrical signals into the deposition volume. The model is presented in Figure 9, where the models in Figure 8 are linear equations, while the adjusted model is a power function after the regression analysis of all samples. The model is expressed as:

$$
y=0.7403 x^{0.9098}
$$

digital measuring system, with $R^{2}$ higher than 0.85 . This means that ISSRMDP could be an indicator for the accurate detection of deposition volume in a typical droplet size range, based on the calibrating model.
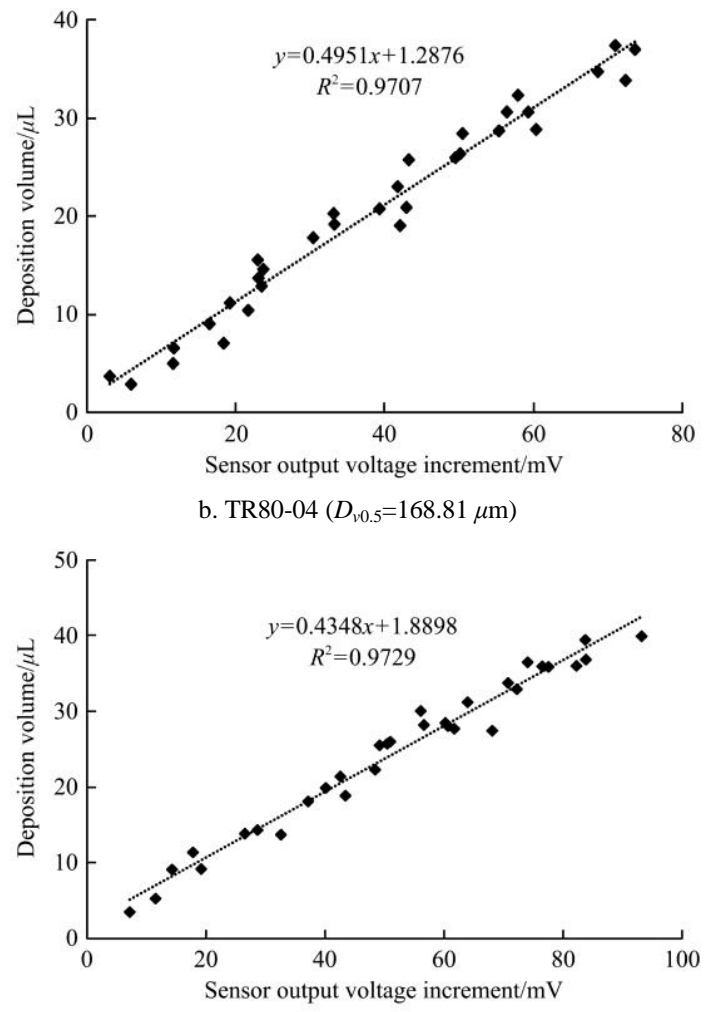

d. $\operatorname{ST110-08~}\left(D_{v 0.5}=207.51 \mu \mathrm{m}\right)$

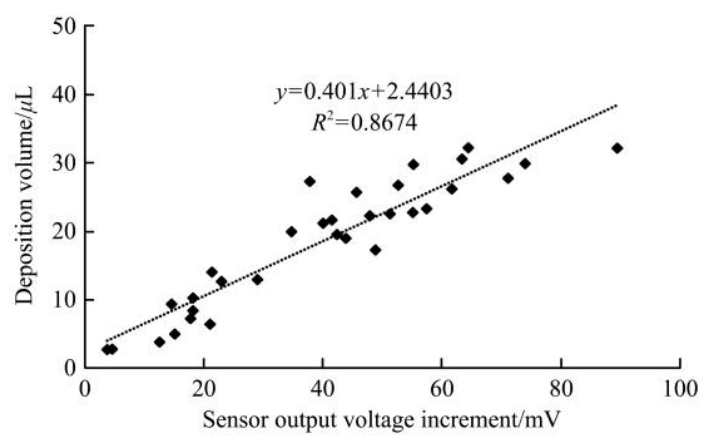

f. IDK120-04 $\left(D_{v 0.5}=396.20 \mu \mathrm{m}\right)$ b. TR80-04 $\left(D_{v 0.5}=168.81 \mu \mathrm{m}\right)$

where, $y$ is deposition volume, $\mu \mathrm{L} ; x$ is the voltage increment from ISSRMDP, $\mathrm{mV}$.

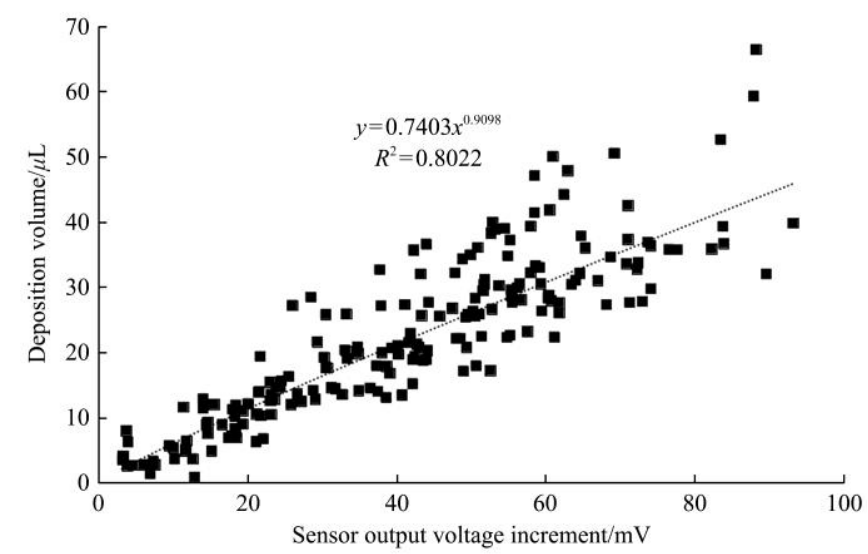

Figure 9 The model for predicting deposition volume based on ISSRMDP 
The determination coefficient $R^{2}$ was 0.8022 , hence the $80.22 \%$ variation in the signal of the developed sensor could be explained by the measurement of the voltage increment, which indicates its application in droplet deposition test is feasible. The determination coefficient was lower than that of the individual test for each nozzle, which can be probably explained by the excessive or smaller size droplets shown in Figures 8a and 8f. In addition, the power regression model demonstrates that when the deposition volume is below $20 \mu \mathrm{L}$, the predicted value is concentrated around the regression line, which means that it can obtain better prediction results in this range.

\subsection{Applying ISSRMDP in the field}

To further explore the applicability of the ISSRMDP, a field experiment was conducted. Droplets number per unit area on WSP were analyzed using professional software, and the deposition volume on leaf sensor was determined via spectrophotometer and real-time measurement system. The result is shown in Table 4. Regarding the deposition volume, ISSRMDP exhibited an optimal performance, as the minimum deviation of deposition volume measured by this sensor system from spectrophotometer method was $0.974 \%$ and the overall deviation was less than $10 \%$.

Table 4 Droplet deposition determined by WPS, spectrophotometer and ISSRMDP

\begin{tabular}{|c|c|c|c|c|c|c|c|}
\hline \multirow{2}{*}{ Value } & \multicolumn{6}{|c|}{ Sample number } & \multirow{2}{*}{ Average } \\
\hline & $\mathrm{S} 1$ & $\mathrm{~S} 2$ & S3 & $\mathrm{S} 4$ & S5 & S6 & \\
\hline $\begin{array}{l}\text { Droplet number on } \\
\mathrm{WSP} / \mathrm{cm}^{-2}\end{array}$ & 8.337 & 22.455 & 49.263 & 62.169 & 12.017 & 11.399 & 27.607 \\
\hline $\begin{array}{l}\text { Deposition volume } \\
\text { measured by } \\
\text { spectrophotometer } / \mu \mathrm{L}\end{array}$ & 3.337 & 3.678 & 9.425 & 12.107 & 2.835 & 2.682 & 5.677 \\
\hline $\begin{array}{l}\text { Deposition volume } \\
\text { predicted by } \\
\text { ISSRMDP/ } \mu \mathrm{L}\end{array}$ & 3.105 & 3.960 & 10.262 & 11.989 & 2.991 & 2.415 & 5.787 \\
\hline $\begin{array}{l}\text { Deviation (absolute } \\
\text { value) } / \%\end{array}$ & 6.952 & 7.667 & 8.880 & 0.974 & 5.502 & 9.955 & I \\
\hline
\end{tabular}

WSP is a conventional method for measuring the droplet number in real-time to compare the spray performance of different application techniques and equipment. Therefore, in the experiment, WSP was introduced to compare the correlation between the real-time predicted value and droplet number. As shown in Table 4 , the average number of droplets in six sample points was $27.6 \mathrm{~cm}^{-2}$. Figure 10 illustrates the deposition curves detected by the three methods. The results indicate that the change in the deposition trends was consistent. Before spraying

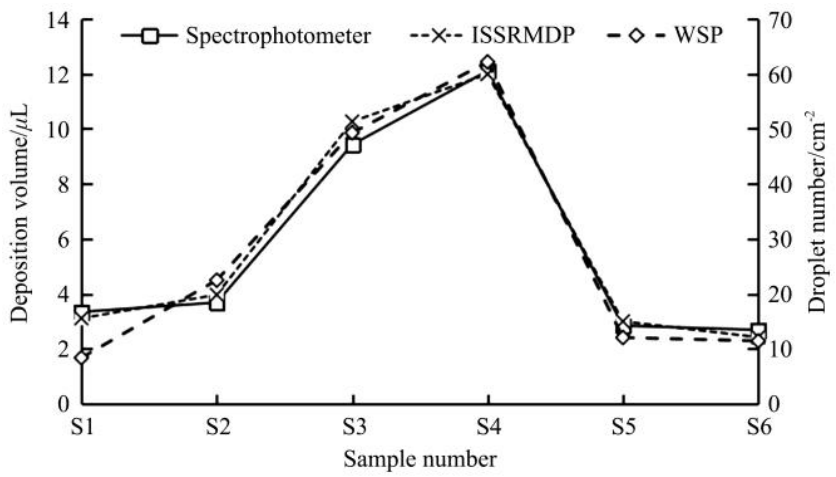

Figure 10 Droplet deposition volume or droplet number at different locations with three methods operation, it is indispensable to test spraying performance of plant protection machinery. Accordingly, the nozzle spacing, spray boom height, spraying parameters were calibrated to ensure a uniform deposition distribution. Based on the system, operator can freely determine the number of sensor nodes according to the sprayer characteristics, and monitor the droplet deposition pattern in real-time. For example, for a large boom sprayer with a boom length of $30 \mathrm{~m}, 60$ sensor nodes can be used simultaneously to obtain the deposition distribution curve in boom direction.

\section{Conclusions}

A novel system was developed based on surface humidity sensors with extreme environment operation and wireless data transmission for the real-time measurement of droplet deposition for agricultural sprayers. An Android application for mobile terminal and the corresponding coordinator were developed, which allowed operators to control multiple sensor nodes simultaneously via the Bluetooth.

Tests using six agricultural nozzles with different droplet sizes were conducted, and the results exhibited an optimal correlation between deposition volume and voltage increment output from sensor $\left(R^{2}=0.8674-0.9729\right)$. In addition, a power regression model based on least squares technique was developed based on all sample data, and the determination coefficient $R^{2}$ was 0.8022 . Also, it was verified that when deposition volume is less than $20 \mu \mathrm{L}$, the novel system exhibits a better ability of prediction.

The proposed system exhibited a better performance than conventional method of measuring tracer concentration in predicting the deposition volume in terms of real-time detection. The minimum deviation of the deposition volume measured by this sensor system from spectrophotometer was $0.974 \%$, and the overall deviation was less than $10 \%$. In addition, the system exhibited a good fitting curve of deposition distribution with droplet number results measured by water sensitive paper method.

The deposition sensing system provides a real-time detection method for testing deposition volume and droplet distribution characteristics. Operators can obtain deposition data in the field by mobile phones, avoiding complicated processing procedures such as collector samples eluting and water-sensitive paper scanning in the laboratory. Further experiments with various spraying chemical mixtures and different sprayers will be carried out to verify the suitability of the system. In addition, more field trials will be conducted to build an optimized calibration model via the analysis of a large amount of data to improve detection accuracy.

\section{Acknowledgements}

The authors acknowledge that this work was financially supported by the National Key Research and Development Project of China (Grant No. 2019YFD1101102-3), Youth Research Fund of Beijing Academy of Agriculture and Forestry Sciences (Grant No. QNJJ202009), Outstanding Scientist Cultivation Project of Beijing Academy of Agriculture and Forestry Sciences (Grant No. JKZX201903), National Natural Science Foundation of China (Grant No. 32071907), and Outstanding Young Talents Projects of Beijing Academy of Agriculture and Forestry Sciences-Research on positioning and control technology and equipment of unmanned vehicles in orchards. Also, Shuaihui Feng, Shufan Chai, Mingjia Zhang and Jiaxing Song's contributions to this experimental work are highly appreciated. 


\section{[References]}

[1] Huang Y, Hoffmann W C, Lan Y, Wu W, Fritz B K. Development of a spray system for an unmanned aerial vehicle platform. Applied Engineering in Agriculture, 2008; 25(6): 803-809.

[2] Chen S D, Lan Y B, Li J Y, Zhou Z Y, Jin J, Liu A M. Effect of spray parameters of small unmanned helicopter on distribution regularity of droplet deposition in hybrid rice canopy. Transactions of the CSAE, 2016; 32(17): 40-46. (in Chinese)

[3] Li L L, He X K, Song J L, Liu Y J, Zeng A J, Liu Y J. Design and experiment of variable rate orchard sprayer based on laser scanning sensor. Int J Agric \& Biol Eng, 2018; 11(1): 101-108.

[4] Dekeyser D, Foqué D, Duga A T, Verboven P, Hendrickx N, Nuyttens D. Spray deposition assessment using different application techniques in artificial orchard trees. Crop Protection, 2014; 64: 187-197.

[5] Gil E, Llorens J, Landers A, Llop J, Giralt L. Field validation of DOSAVINA, a decision support system to determine the optimal volume rate for pesticide application in vineyards. European Journal of Agronomy, 2011; 35(1): 33-46.

[6] Cunha J P A R, Farnese A C, Olivet J J. Computer programs for analysis of droplets sprayed on water sensitive papers. Planta Daninha, 2013; 31(3): 715-720

[7] Wen Y, Zhang R R, Chen L P, Huang Y B, Yi T C, Xu G, et al. A new spray deposition pattern measurement system based on spectral analysis of a fluorescent tracer. Computers and Electronics in Agriculture, 2019; 160:14-22.

[8] Gao S, Wang G, Zhou Y Y, Wang M, Yang D B, Yuan H Z, et al. Water soluble food dye of allura red as a tracer to determine the spray deposition of pesticide on target crops. Pest Management Science, 2019; 75: 2592-2597.

[9] Qiu J, Zheng J Q, Zhou H P. Summarization of droplet size determination and treatment methods. Forestry Machinery \& Woodworking Equipment, 1999; 27(7): 10-12. (in Chinese)

[10] Xue Feng. Application of digital image processing in droplet key parameters measurement. Master dissertation. Beijing: China Agriculture University, 2005; 2. 24 p.

[11] Justin J N, Forster W A. Due diligence required to quantify and visualise agrichemical spray deposits using dye tracers. Crop Protection, 2019; 115 : 92-98.

[12] Wang X N, He X K, Song J L, Wang Z C, Wang CL, Wang S L. Drift potential of UAV with adjuvants in aerial applications. Int J Agric \& Biol Eng, 2018; 11(5): 54-58.

[13] Liop J, Gil E, Llorens J, Gallart M, Balsari P. Influence of air-assistance on spray application for tomato plants in greenhouses. Crop Protection, 2015; 78: 293-301

[14] Cross J V, Murray R A, Ridout M S, Walklate P J. Quantification of spray deposits and their variability on apple trees. Aspects of Applied Biology (United Kingdom), 1997; 48: 217-224.

[15] Nairn J J, Forster W A. Photostability of pyranine and suitability as a spray drift tracer. New Zealand Plant Protection, 2015; 68: 32-37.

[16] Gil Y, Sinfort C. Emission of pesticides to the air during sprayer application: A bibliographic review. Atmospheric Environment, 2005; 39(28): 5183-5193.

[17] Gil E, Escolà A, Rosell J R, Planas S, Val L. Variable rate application of plant protection products in vineyard using ultrasonic sensors. Crop Protection, 2007; 26(8): 1287-1297.

[18] Cruvinel P E, Vieira S R, Crestana S, Minatel E R, Mucheroni M L, Neto A T. Image processing in automated measurements of raindrop size and distribution. Computers and Electronics in Agriculture, 1999; 23(3): 205-217.

[19] Cunha M, Carvalho C, MarcalA R S. Assessing the ability of image processing software to analyse spray quality on water-sensitive papers used as artificial targets. Biosystems Engineering, 2012; 111(1): 11-23.

[20] Salyani M, Zhu H, Sweeb R D, Pai N. Assessment of spray distribution with water-sensitive paper. Agricultural Engineering International: The CIGR e-journal, 2013; 15(2): 101-111.

[21] Salyani M, Serdynski J. Development of a sensor for spray deposition assessment. Transactions of the ASAE, 1990; 33(5): 1464-1469.

[22] Zhang R R, Wen Y, Yi T C, Chen L P, Xu G. Development and application of aerial spray droplets deposition performance measurement system based on spectral analysis technology. Transactions of the CSAE, 2017; 33(24): 80-87. (in Chinese)

[23] Musiu E M, Qi L, Wu Y. Spray deposition and distribution on the targets and losses to the ground as affected by application volume rate, airflow rate and target position. Crop Protection, 2019; 116: 170-180.

[24] Pergher G, Gubiani R, Cividino S R S, Dell'Antonia D, Lagazzio C. Assessment of spray deposition and recycling rate in the vineyard from a new type of air-assisted tunnel sprayer. Crop Protection, 2013; 45: 6-14.

[25] Paolo B, Marco G, Paolo M, Matta F, Miranda F A. Assessing the influence of air speed and liquid flow rate on the droplet size and homogeneity in pneumatic spraying. Pest Management Science, 2019; 75(2): 366-379.

[26] Zhu H, Salyani M, Fox R D. A portable scanning system for evaluation of spray deposit distribution. Computers and Electronics in Agriculture, 2011; 76(1): 38-43. 